Artigo Original

Gestão em Saúde

\title{
DIAGNÓSTICO DO MANEJO INTRAESTABELECIMENTO DOS RESÍDUOS DE SERVIÇOS DE SAÚDE DE UMA UNIDADE BÁSICA DE SAÚDE DO EXTREMO SUL CATARINENSE
}

Management diagnosis within the establishment of the health service waste a basic unit do extremo sul catarinense health

Clarice Costa Custódio ${ }^{1}$, Mario Ricardo Guadagnin²

${ }^{1}$ Graduanda em Biomedicina, Universidade do Extremo Sul Catarinense.

${ }^{2}$ Universidade do Extremo Sul Catarinense.

Endereço para correspondência:

Clarice Costa Custódio

Rua Caetano Lummertz, 1104, Araranguá, SC, Brasil.

Email: claricecostacustodio@hotmail.com 


\section{Resumo}

O manejo dos resíduos sólidos de serviços de saúde é um assunto que evolui no decorrer do tempo. Este tema está regulamentado por uma legislação própria expedida pela ANVISA e CONAMA, rígida e que conta com órgãos fiscalizadores. A cobrança aos estabelecimentos geradores dos resíduos de serviços de saúde, principalmente no que diz respeito ao manejo intraestabelecimento, tem aumentado a necessidade de gerenciar o processo. As unidades básicas de saúde são estabelecimentos que visam tratar a saúde da comunidade, desta forma, torna-se um local gerador de resíduos sólidos de serviços de saúde, o que acarreta a necessidade de possuir um plano de gerenciamento efetivo. A pesquisa teve como objetivo diagnosticar o manejo intraestabelecimento dos resíduos sólidos de serviço de saúde em uma unidade básica de saúde do município de Araranguá, SC. Tal processo foi analisado em todas as suas subdivisões (segregação, acondicionamento, transporte interno, armazenamento interno, transporte externo, armazenamento externo e o tratamento interno), como são realizados e a sua adequação às resoluções vigentes. A verificação foi realizada mediante observação das etapas que seguiram um check list e foram analisadas de acordo com a legislação vigente, em especial a RDC 306/2004 da ANVISA. Desta forma a pesquisa teve cunho qualitativo e descritivo, por meio de percepção visual. Observou-se, no período de diagnóstico, irregularidades no processo de manejo dos resíduos gerados. Todos os resultados foram descritos de forma a transmitir a realidade percebida no local e relatar pontos passíveis de melhorias e atendimentos às legislações vigentes. Desta forma, este trabalho buscou, ainda, expor a importância de realizar corretamente as etapas do processo de manejo dos resíduos sólidos de serviços de saúde intraestabelecimento, caracterizando um gerenciamento eficaz dos mesmos. Seguir as normas estabelecidas proporciona boas condições de trabalho, integridade da saúde como um todo e a preservação do meio ambiente. No entanto o estudo aponta para incongruências e a necessidade da promoção de capacitação dos colaboradores da unidade, bem como da própria gestão em saúde do município.

Palavras-chave: Resíduos; Saúde; Gerenciamento; Manejo. 


\section{Abstract}

The management of solid residues of health services is a subject that evolves over time. This theme is regulated by a proper and strict legislation issued by ANVISA and CONAMA, which has regulatory agencies. Charging the waste generators health service establishments, especially in regard to the inside establishment management, has increased the need to manage the process. The basic health units are establishments that aim to treat the health of the community. Thus, they become a generator place of solid residues of health services, which entail the need to have an effective management plan. The research aimed to diagnose the inside establishment management of solid waste health service in a basic health unit in the city of Araranguá-SC. This process was analysed in all its subdivisions (segregation, packaging, internal transport, internal storage, external transport, external storage and internal treatment) as they are operated and the adequacy of the existing resolutions. The verification was carried out by observing the steps that followed a checklist and were analysed according to the current legislation, in particular the RDC 306/2004 of ANVISA. In this way the research had qualitative and descriptive approach through visual perception. It was observed in the diagnostic period, irregularities in the management process of the produced residues. All results were described in order to transmit the perceived reality in place and report points passive of improvement and regards for current legislations. By this way, this study sought to explain yet the importance of realizing properly the steps of the management process of solid residues of inside establishment's health services, featuring an effective management about them. Following the established standards provides good working conditions, health integrity as a whole and the environment preservation. However, the study points to inconsistencies and the need for promotion of training employees of the health unit, as well the city's own health management.

Keywords: Residues; Health; Management; Establishments. 
Com o aumento do número populacional aderido aos novos costumes consumistas, juntamente com a industrialização e a uma melhoria do poder aquisitivo, amplia-se a preocupação com a problemática ambiental, pois a geração de resíduos sólidos tem acompanhado esse crescimento¹.

Segundo Domenéch ${ }^{2}$, resíduo é todo o produto gerado e indesejado, fruto de atividade humana ou de qualquer ser vivo. Já os resíduos sólidos são de origem antropogênica, pois são gerados exclusivamente pelo homem ${ }^{3}$. Para a Associação Brasileira de Normas Técnicas (ABNT), na Norma Brasileira (NBR) 10004/87, Resíduo sólido é "produto resultante de atividades da comunidade, de origem industrial, domiciliar, hospitalar, radioativa, comercial, agrícola e de varrição"3.

Segundo a Política Nacional de Resíduos Sólidos, pela lei 12305 de $2010^{4}$, os resíduos sólidos são classificados de duas maneiras: quanto à sua origem e quanto à sua periculosidade.

Um dos agentes de degradação ambiental que possui um nível considerável de periculosidade são os resíduos sólidos, gerados em estabelecimentos da área da saúde¹. A ABNT, NBR 12807 de 1993, descreveu que resíduos sólidos de serviços de saúde (RSSS) são os resíduos resultantes das atividades exercidas por estabelecimentos prestadores de serviços da saúde ${ }^{5}$.

Com esta visão, os RSSS possuem como melhor opção de tratamento o seu gerenciamento, que visa diminuir a sua produção exacerbada e encaminhar o montante produzido a uma destinação correta, de maneira segura, prezando sempre pela proteção dos profissionais envolvidos em todas as fases do processo, pela manutenção da saúde pública e dos recursos ambientais ${ }^{6}$. No entanto, para que haja bons resultados neste processo de gerenciamento, requer-se investimento na capacitação de todos os profissionais envolvidos na área da saúde. A capacitação dos profissionais tem o intuito de orientar como destinar com resíduos, bem como manusear de forma correta e a utilização de equipamentos de proteção ${ }^{7}$. Tal capacitação é descrita ainda pela NBR 12809 


\section{Artigo Original}

\section{Gestão em Saúde}

de 1993, que descreve sobre o conhecimento dos funcionários sobre o sistema de identificação dos resíduos, para que os mesmos possam segregá-los ${ }^{8}$.

Os RSSS, principalmente representados pelos materiais perfurocortantes, quando destinados de maneira incorreta, tornam-se uma importante fonte de infecções ${ }^{9}$.

Desta maneira, estudar o gerenciamento de RSSS é de suma importância, seja para desenvolver técnicas, ter um levantamento da situação ou corrigir possíveis erros de processo. Principalmente para municípios de pequeno porte, saber a situação do gerenciamento de seus resíduos intraestabelecimento de suas unidades básicas de saúde é extremamente importante. Desta forma, o município obtém o conhecimento necessário para saber onde agir, visando solucionar erros, e, assim, com o crescimento da região, acompanhar o desenvolvimento correto do processo de gerenciamento de resíduos da saúde das suas unidades de saúde.

\section{Resíduos sólidos de serviços da saúde}

Resíduos sólidos de serviços de saúde geralmente são caracterizados erroneamente, uma vez que muitas pessoas consideram apenas os resíduos provenientes de hospitais e clínicas médicas como a única fonte geradora de resíduos de serviços da saúde, denominando-o de "lixo hospitalar". Porém, existe uma gama de estabelecimentos que produzem essa classe de resíduos ${ }^{10}$.

De acordo com a resolução no 358 de 29 de abril de 2005 do CONAMA, resíduos de serviços da saúde são aqueles que resultam de atividades exercidas em prestadores de serviços a saúde, que, por suas características, necessitam de processos de manejo diferente dos demais resíduos ${ }^{11}$.

Para Fortes ${ }^{12}$, os resíduos de serviços de saúde são fracionados, onde cada fração é composta por diferentes estabelecimentos geradores. Estes estabelecimentos então geram resíduos que compreendem materiais como perfurocortantes contaminados, peças anatômicas, produtos químicos, papel de escritório, restos de alimentos, entre outros ${ }^{12}$. 
Artigo Original

Gestão em Saúde

\section{Classificação dos resíduos de serviços da saúde}

Os resíduos de serviços de saúde possuem uma classificação de acordo com a resolução ANVISA 306/2004, onde se subdividem em cinco grupos: A, são aqueles que possuem agentes biológicos; $B$, são os de natureza química; $C$, são os radioativos; D, se caracterizam por serem os comuns; E, os perfurocortantes ${ }^{13}$.

\section{Gerenciamento de resíduos sólidos de serviços da saúde}

Implantar, cumprir e melhorar o sistema que permite dar a destinação correta aos resíduos gerados pelas atividades humanas tem sido um desafio imposto à sociedade atual. $\mathrm{O}$ desenvolvimento populacional aumentou e com isso a geração de resíduos também. Esta problemática não pode ser ignorada pelo tamanho da sua gravidade ${ }^{14}$.

Desta maneira, administrar a destinação dos resíduos torna-se uma questão de suma importância para a preservação da saúde populacional e ambiental. O gerenciamento, além de acompanhar o processo dos resíduos, tem o poder de reduzir significativamente o volume de resíduos gerados. No entanto, os profissionais devem ser conscientizados e treinados para esse processo ${ }^{15}$.

Com esta visão, criar um plano de gerenciamento de resíduos é essencial ao estabelecimento gerador. Tal plano visa reduzir os riscos à saúde da população. Desta maneira, as características de cada estabelecimento devem ser levadas em consideração para a elaboração do plano ${ }^{3}$.

De acordo com a RDC 306 de 2004 da ANVISA, o programa de gerenciamento de resíduos de serviço de saúde subdivide o processo de manejo intraestabelecimento em: segregação, acondicionamento, identificação, transporte interno, armazenamento temporário, tratamento e armazenamento externo. Uma vez que, segundo a mesma RDC, o manejo dos RSSS é entendido como ação de gerenciar os resíduos de acordo com seus aspectos intra e extra estabelecimento, desde a geração até a deposição final ${ }^{13}$.

A primeira etapa do processo do manejo intraestabelecimento é a segregação, que se caracteriza por ser a separação dos resíduos no momento 


\section{Artigo Original}

\section{Gestão em Saúde}

e no local onde foram gerados. Este processo leva em conta as características físicas, químicas, biológicas, sua espécie, estado físico e classificação. Esta segregação deve ser feita em embalagens corretas, ou seja, o processo do acondicionamento, que consiste em embalar os resíduos gerados, de acordo com suas características em recipientes impermeáveis e resistentes a punctura, ruptura e vazamentos. A capacidade dos recipientes de acondicionamento deve ser compatível com o fluxo de geração diária de cada tipo de resíduo ${ }^{13}$.

A identificação é o processo que permite o reconhecimento dos resíduos que estão embalados. Esta etapa é crucial para que haja um correto manejo do material. A identificação deve ser feita não só nos recipientes internos, como também nos recipientes de transporte externo e nos locais de armazenamento. Esta identificação é subdividida em grupos de resíduos, sendo eles: A) devem ser identificados com o símbolo de substância infectante, com rótulos de fundo branco, desenhos e contorno em preto; B) devem ser identificados com 0 símbolo de risco associado, discriminação de substância química e frases de risco; C) são identificados pelo símbolo radiação ionizante, em rótulos de fundo amarelo e contornos pretos, acrescido da expressão "Rejeito Radioativo"; D) Resíduo comum; E) identificados pelo símbolo de substância infectante, com rótulos de fundo branco, com desenhos e contornos em preto e escrito "Resíduo Perfurocortante"13.

O processo continua com o transporte interno, que é o processo onde os resíduos são encaminhados ao local de armazenamento temporário ou à coleta externa. O translado interno dos resíduos tem que seguir um protocolo, onde deve ser realizado em sentido único, com roteiro e horários definidos, para que não coincida com os procedimentos diários. Este transporte é responsável por levar os resíduos segregados, acondicionados e identificados até o local do armazenamento temporário, sendo que este ambiente é onde ocorre a guarda temporária destes resíduos em um local próximo aos pontos de geração, visando a coleta dentro do estabelecimento e otimizar o deslocamento entre os pontos geradores e o ponto de apresentação à coleta externa ${ }^{13}$.

O estabelecimento pode contar com o tratamento interno, que é o primeiro tratamento dos resíduos, feito no próprio estabelecimento gerador, obedecendo às condições de segurança. Para que ele seja feito, deve-se ter 


\section{Artigo Original}

\section{Gestão em Saúde}

licenciamento ambiental e este local será passível de fiscalização e de controle pelos órgãos de vigilância sanitária e de meio ambiente. Este processo visa reduzir ou eliminar o risco de contaminação ocupacional e ambiental, por meio de modificações nas características de risco dos resíduos ${ }^{13}$.

O tratamento desses resíduos, intraestabelecimento, pode se dar por autoclavagem, microondas, desinfecção química e incineração ${ }^{16}$.

Por fim o armazenamento externo é o local em que os resíduos irão permanecer até a coleta externa ${ }^{13}$.

\section{Unidades básicas de saúde e o gerenciamento dos RSS}

A atenção básica à saúde tem como principal local de atuação as Unidades Básicas de Saúde (UBS). Estas, por sua vez, atendem a uma população específica que se encontra em um local definido ${ }^{17}$.

Desta forma, as UBS também geram resíduos sólidos de serviços de saúde, que de acordo com Silva e Hoppe ${ }^{18}$, apesar de ser em um volume proporcionalmente menor a todos os outros resíduos sólidos gerados pela comunidade, possui um grau de periculosidade maior, por se tratar de uma fonte de propagação de doenças, quando gerenciados de maneira inadequada ${ }^{18}$.

Além de pôr em risco a saúde da população e do meio ambiente, existe a preocupação com a saúde ocupacional. Desta forma existe uma atenção voltada à segregação e ao manejo correto dos resíduos para preservar a integridade do trabalhador, seja ele da área da saúde ou responsável pelos serviços de limpeza ${ }^{19}$.

Acidentes biológicos tendo como precursor materiais perfurocortantes representam um grande risco a saúde do trabalhador, o expondo à possibilidade de contrair o vírus como o da hepatite $\mathrm{B}$ e $\mathrm{C}$, e o próprio Vírus da Imunodeficiência Humana (HIV) 20 .

Segundo estudo realizado em 21 Unidades de Saúde da Família no município de Maranguape - CE, nenhuma enfermeira, ao ser questionada sobre as dificuldades na realização do exame Papanicolau, citou o descarte de resíduos como um empecilho para a realização. No entanto, em $100 \%$ das vezes o descarte dos resíduos ocorreu de forma inadequada. Desta forma, torna-se 


\section{Artigo Original}

\section{Gestão em Saúde}

notável a falta de preocupação e consciência destas profissionais sobre o seu ato ${ }^{21}$.

De acordo com Almeida et al. (2009), os materiais perfurocortantes, em sua maioria, são acondicionados de forma correta nas unidades de saúde. Porém, acabam sendo misturados com materiais do grupo A e $D$, por questões de conhecimento dos profissionais, que acabam por não fazer as capacitações, como também pela falta de embalagens adequadas ${ }^{22}$.

\section{MÉTODO}

Para o decorrer do estudo foi solicitada a autorização do desenvolvimento da pesquisa, por meio de Carta de Aceite, em uma Unidade Básica de Saúde, carta esta submetida à Secretaria Municipal de Saúde, para autorização. O primeiro andamento tomado foi o contato primário com a equipe de saúde do município para o agendamento das visitas ao local da realização da pesquisa. Para obter os dados necessários referentes ao diagnóstico da situação dos resíduos sólidos de serviços de saúde foi realizada uma pesquisa descritiva qualitativa. Tal modalidade de pesquisa caracteriza-se como um estudo que visa projetar um futuro diante das respostas encontradas. A importância desta pesquisa está ligada à resolução de problemas através da análise e descrição de fatos observados ${ }^{23}$.

Sendo assim, os dados para a análise foram coletados por meio de visitas à Unidade Básica de Saúde em questão, onde houve percepção visual dos pontos observados. As análises que foram realizadas estão de acordo com o que preconiza a legislação vigente, no que diz respeito ao manejo de resíduos sólidos de serviços de saúde, neste caso a Resolução n 306/2004 da ANVISA.

Para a execução da observação "in loco", de acordo com a resolução da ANVISA, foram analisados pontos como: segregação, acondicionamento, identificação, transporte interno e armazenamento temporário, transporte externo, armazenamento externo e tratamento interno.

Ao final dos levantamentos, os dados foram apresentados de forma descritiva aos responsáveis da secretaria de saúde, para as possíveis 
Artigo Original

Gestão em Saúde

adequações de acordo com o preconizado pela resolução da Vigilância Sanitária.

\section{APRESENTAÇÃO E ANÁLISE DOS DADOS}

O estudo decorreu em uma Unidade Básica de Saúde do Extremo Sul Catarinense, que realiza atendimentos médicos, de enfermagem e odontológicos. A unidade disponibiliza à população consultas médicas, procedimentos simples como curativos, aplicação de vacinas segundo 0 calendário vacinal, testes rápidos, consultas odontológicas com seu respectivo tratamento e o exame preventivo do câncer de colo uterino. Por meio destas atividades o estabelecimento gera resíduos comuns, perfurocortantes, biológicos, químicos.

A unidade abrange uma área de, em média, quatro mil moradores, os quais são atendidos por uma equipe composta por quatorze funcionários.

\section{Apresentação da Unidade Básica de Saúde}

A referida unidade está instalada em uma casa, a qual foi adaptada às necessidades de uma Unidade Básica de Saúde, reformada e ampliada em 2014. As instalações são constituídas por quatorze setores (sala de espera, recepção, sala de vacina, sala de triagem, sala de procedimentos, farmácia, sala de armazenamento temporário, sala de utilidades, cozinha, consultório de enfermagem, consultório médico, consultório odontológico, auditório/sala das agentes comunitárias de saúde e banheiros).

\section{Análise dos dados obtidos referentes ao manejo}

De acordo com o que foi observado durante a visita técnica, tendo em vista o check list e a RDC 306/2004 da ANVISA, vários itens foram pontuados e serão descritos qualitativamente por área específica componente do manejo intraestabelecimento. 
Artigo Original

Gestão em Saúde

Segregação

$\mathrm{Na}$ unidade analisada foram observadas algumas segregações de resíduos de maneira incorreta, tendo como base a RDC 306/2004 da ANVISA.

Resíduos do Grupo A

$\mathrm{Na}$ visita realizada, foi observado que itens como luvas, algodão usado e bandagem de testes rápidos foram vistos acondicionados em locais indevidos. Luvas, que poderiam estar contaminadas, estavam presentes em lixo comum. Da mesma forma, algodão e bandagem de testes rápidos foram encontrados na caixa de coleta de perfurocortantes.

Resíduos do Grupo B

Deveria haver na unidade um local para o descarte deste material, porém na visita não foi encontrado. Com isso, resíduos como a bandagem do teste rápido e medicamentos foram vistos em recipientes de coletas não adequados. A bandagem que contém gotas de um reagente químico, por exemplo, foi encontrada na caixa de perfurocortantes.

Não só isso, medicamentos foram encontrados segregados como resíduo comum.

Resíduos do Grupo C

A unidade não gera esse tipo de resíduo.

Resíduos do Grupo D

$\mathrm{Na}$ resolução não há obrigatoriedade sobre a separação destes resíduos, porém, é do saber comum que poderia existir na unidade uma segregação destes materiais com uma visão voltada para a reciclagem. $\mathrm{Na}$ cozinha da unidade pode ser observado resíduo orgânico misturado com resíduo comum. Outro ponto no que se refere a resíduos comuns é que luvas que não 
Artigo Original

Gestão em Saúde

entraram em contato com fluidos corporais ou materiais biológicos podem ser segregadas como resíduo comum.

Resíduos do Grupo E

Este é o material em que os funcionários mais tem cuidado na hora de segregar. Não foi encontrado erro na segregação destes materiais, no entanto, como já apresentado, o local de acondicionamento dos mesmos foi um dos locais que mais apresentou resíduos de outros grupos, segregados de maneira incorreta.

Essa segregação de maneira errônea pode gerar problemas graves, como descrito por Vieira ${ }^{24}$. Tendo isso em vista, quatro pontos principais podem ser levantados quando há um debate sobre a importância em segregar os resíduos de maneira correta. O primeiro ponto é a contaminação de resíduos comuns por contato com resíduos contaminados, o que acarreta o segundo ponto, que é o aumento da complexidade do tratamento dos resíduos visando a destinação final correta. Consequentemente o terceiro ponto surge, que é o aumento do gasto para o tratamento destes resíduos, caso não ocorra a percepção de que um resíduo foi segregado de maneira incorreta pode ainda ocorrer o quarto ponto, que é a poluição ambiental25.

Se ocorresse a correta segregação não haveria contaminação de resíduos e diminuiria o volume de resíduos contaminados, o que traria uma economia aos cofres públicos e principalmente haveria a manutenção da qualidade ambiental.

Acondicionamento

Segundo a RDC 306/2004 da ANVISA, todas as lixeiras devem ser de pedal, para que o trabalhador não seja exposto a riscos de acidentes e infecção. $\mathrm{Na}$ mesma resolução é descrito que as lixeiras devem possuir tampa ${ }^{13}$. $\mathrm{Na}$ unidade visitada pôde ser notado que nem todas as lixeiras seguiam este ponto da resolução. 
Outro ponto que foi encontrado de irregularidade foi que resíduos infectantes devem ser acondicionados em embalagens branco leitosas, o que não é a realidade da unidade. Todas as lixeiras de resíduos infectantes estavam com embalagens pretas, de um material não considerado resistente a puncturas e extravasamento. No consultório médico, local onde ele trabalha com o afastador de língua, este material, que pode ficar contaminado, foi descartado como resíduo comum, onde o acondicionamento é uma embalagem preta comum.

Vale lembrar que, segundo a RDC 306/2004 da ANVISA, os recipientes de acondicionamento de perfurocortantes devem ser descartados quando atingirem 2/3 do seu volume total, sendo que não é permitido o esvaziamento $e$ reaproveitamento ${ }^{13}$. Quanto a isso, a unidade não apresentou irregularidade, uma vez que nenhum dos recipientes de descarte de perfurocortantes estava com mais de $2 / 3$ do seu volume ocupado.

\section{Identificação}

Segundo a RDC 306/2004 da ANVISA, as embalagens onde os resíduos são acondicionados devem ser identificadas com símbolos, de forma padronizada, para que todos que entrem em contato saibam com o que estão lidando. Sendo que a resolução ainda dispõe que não só as embalagens mas os recipientes de segregação (lixeiras) também devem ser identificados com o mesmo símbolo. E por fim, deve-se ter cuidado para que a embalagem do acondicionamento não tape a identificação do recipiente ${ }^{13}$.

Resíduos do Grupo A

Irregularidades foram observadas na unidade, uma vez que as lixeiras de resíduos infectantes estavam sem a identificação na embalagem e no recipiente coletor.

Porém, na sala de armazenamento temporário o recipiente coletor estava corretamente identificado e rotulado. Possuía embalagem branco leitosa também identificada com o símbolo de substância infectante. No entanto, dentro 


\section{Artigo Original}

\section{Gestão em Saúde}

desta embalagem branco leitosa são colocadas todas as embalagens contendo resíduos segregados na unidade, sem as devidas identificações. Desta forma, pode ser que haja só resíduos infectantes dentro, bem como pode ter resíduos comuns, não há maneira de saber. E quando a empresa coleta, e a identificação é de resíduo infectante, como é a embalagem do armazenamento temporário, eles não vão abrir a embalagem e separar os resíduos, sendo assim, tudo que está naquela embalagem será tratada como resíduo infectante.

Resíduos do Grupo B

Foi observado na unidade que a bandagem de teste rápido, que é contaminada com reagente químico, foi descartada no recipiente para resíduos perfurocortantes, bem como medicamentos descartados em recipiente para resíduos comuns.

Outro ponto levantado na análise da unidade foi que no consultório médico havia apenas um recipiente de segregação, sem identificação, onde era descartado resíduo comum e resíduos infectantes, como os afastadores de língua.

Resíduos do Grupo C

A unidade não gera esse tipo de resíduo.

Resíduos do Grupo D

Apesar de resíduos comuns não produzirem algum risco a sociedade, os recipientes em que são segregados devem ser identificados. Na unidade visitada foi observado que mais da metade dos recipientes de segregação não possuíam identificação.

Resíduos do Grupo E 
Artigo Original

Gestão em Saúde

Foi observado, na Unidade Básica de Saúde, que todos os recipientes e embalagens deste grupo estavam de forma correta.

\section{Transporte Interno}

O transporte interno dos resíduos acontece sem seguir um protocolo. As embalagens contendo os resíduos segregados são manejadas pela profissional de serviços gerais, ou, à maneira que enchem, pelos próprios profissionais de saúde, que os recolhem de acordo com os seus cronogramas diários de trabalho, bem como na medida em que a embalagem enche. Desta forma, não seguem horários definidos, roteiro ou um único sentido.

Com isso, de acordo com a RDC 306/2004 da ANVISA, há um aumento da possibilidade de contaminação de roupas que estejam sendo distribuídas, neste caso os lençóis das macas, e dos alimentos que possam estar sendo manipulados na unidade. Pode-se ainda contaminar medicamentos que estejam sendo distribuídos na farmácia da unidade e propiciar uma contaminação caso esse manejo ocorra em um período com maior fluxo de pessoas ${ }^{13}$.

\section{Armazenamento Temporário}

O armazenamento interno da unidade analisada se dá em uma sala, dentro do estabelecimento. As embalagens contendo os resíduos segregados, por seu pequeno volume, são acondicionadas em um tonel plástico, o que, segundo a RDC 306/2004 da ANVISA, é denominado como recipiente coletor. A resolução descreve que a sala de armazenamento temporário deve ter paredes lisas e laváveis, o que constatamos que na unidade possui. A RDC ainda dispõe sobre a identificação da porta da sala, que observamos não possuir identificação ${ }^{13}$.

Também foi constatado na unidade a proximidade da sala de armazenamento temporário com os locais de segregação, bem como a facilidade em a empresa terceirizada pela coleta fazer a retirada das embalagens. Pontos estes positivos, pois estão de acordo com a resolução. 
Artigo Original

Gestão em Saúde

Tratamento Interno

A unidade realiza como tratamento interno o processo de autoclavagem. Processo esse realizado com alguns resíduos odontológicos, bem como nos frascos de vacina, para depois serem descartados como perfurocortantes. As autoclaves utilizadas ficam na sala de procedimento.

\section{Armazenamento Externo}

Ao realizar uma análise do espaço físico externo da unidade, foi constatado um possível local que poderia estar servindo para armazenamento externo. O uso deste espaço facilitaria ainda mais a retirada destas embalagens, diminuindo assim a chance de contaminação.

Caso este local encontrado não seja suficiente para a demanda, observamos que a unidade possui um pátio em um tamanho considerável, o qual poderia comportar um espaço para o armazenamento externo. Porém, para que isso viesse a ser uma realidade, deveria ser construído uma sala, o que dependeria de verba financeira para a construção e posteriormente manutenção.

\section{Biossegurança}

Os funcionários que lidam com o processo de manejo dos resíduos de serviços de saúde utilizam como EPI's o jaleco e luvas de látex descartáveis. As mulheres estavam de cabelo preso. Como a unidade foi visitada no inverno, todos estavam de calça comprida e sapato fechado.

\section{CONSIDERAÇÕES FINAIS}

O processo de manejo dos resíduos de serviços de saúde intraestabelecimento, desde as suas fases inicias até as finais, se mostra como ponto importante em vários quesitos. Desde a preservação ou degradação ambiental, preservação da saúde humana, risco de acidente de trabalho com risco aos colaboradores da unidade básica de saúde ou eventual contaminação 


\section{Artigo Original}

\section{Gestão em Saúde}

do próprio usuário. Visando promover esse processo de manejo de forma correta, ampliou-se nos últimos tempos a importância do PGRSS, o qual hoje é um dos documentos necessários para que possa ser emitido o alvará de funcionamento da unidade.

A definição de estratégias e diretrizes que orientam todo e qualquer profissional atuante com uma fonte geradora de resíduo, independente do seu grau de risco e periculosidade, requer um olhar atento, dedicado, detalhado com definições desde o reconhecimento das fontes geradoras, da classificação do resíduo, segregação e acondicionamento correto, do manejo interno, de possibilidades de tratamento interno e disposição ambientalmente adequada. Essas são etapas que compõe um PGRSS, pois definem procedimentos metodológicos e tecnológicos onde o primeiro olhar é para preservar a saúde de quem opera, trabalha, gera o resíduo e consequentemente o ambiente como um todo.

Ao observar os procedimentos internos dos colaboradores de uma Unidade Básica de Saúde e confrontar questionamentos sobre definições de políticas de gestão e gerenciamento para todas as unidades do município, observou-se a inexistência do PGRSS. É recomendável sanar esta lacuna, pois a discussão de sua elaboração seguindo as diretrizes estabelecidas segundo as normas técnicas possibilitará um empoderamento e capacitação para aperfeiçoar e melhorar o gerenciamento existente.

Tendo em vista a RDC 306/2004 da ANVISA como sendo a legislação regulamentadora deste processo, contando com a complementação de outras legislações como a RDC 358, as normativas da ABNT e as resoluções do CONAMA, neste trabalho foram avaliados todos os pontos pertinentes ao processo do manejo intraestabelecimento dos RSS em uma Unidade Básica de Saúde, onde foram observados vários equívocos de procedimentos e de compreensão das diretrizes e princípios normativos, assim como alguns acertos.

Nenhum dos processos (segregação, acondicionamento, identificação, transporte interno e armazenamento temporário) segue as resoluções de maneira integral. Os motivos percebidos na equipe de trabalho desta unidade que condicionam esse comportamento são: falta de tempo, desinteresse e desconhecimento. Apenas um tratamento interno, que é feito com a autoclave, 


\section{Artigo Original}

\section{Gestão em Saúde}

para inertizar frascos de acondicionamento de vacina, é realizado antes da disposição junto com perfurocortante.

Caso ocorresse a correta segregação, não haveria contaminação cruzada de resíduos e diminuiria o volume de resíduos contaminados, o que traria uma economia aos cofres públicos e principalmente haveria a manutenção da qualidade ambiental. Porém, os trabalhadores da unidade e as gestoras não apresentaram um pensamento de preocupação com as finanças públicas, ambiental e com relação aos riscos.

Os funcionários se apresentaram coerentes ao uso dos EPI's durante $o$ processo de manejo dos RSS.

Foi constatado também a falta de conhecimento sobre a temática dos RSS, o que está prejudicando o processo em todos os quesitos. Com relação aos funcionários, um ponto importante observado foi a preocupação dos mesmos com os acidentes de trabalho com perfurocortantes, sendo os resíduos do grupo $\mathrm{E}$ os que obtém a maior atenção dos funcionários.

Desta maneira, ficou nítida a necessidade de um profissional especializado na área para promover educação continuada, oficinas, reflexão e atividades práticas aos trabalhadores da Unidade Básica de Saúde, para que a partir de então possa se ter um cuidado maior com todas as fases do manejo por parte dos funcionários. Com isso, o aumento da cobrança deve ser iniciado também, até que se tenha por hábito fazer o correto.

Outra proposta interessante ao município seria a elaboração de uma semana voltada à saúde no trabalho, de modo a haver um olhar voltado aos riscos associados a um manejo indevido ou a falta de conhecimento sobre o assunto. Este evento deveria envolver todos os funcionários da rede de saúde, sem hierarquia.

\section{REFERÊNCIAS}

1 - Coelho H. Gerenciamento de resíduos sólidos de serviços de saúde. Boletim de Pneumologia Sanitária, Rio de Janeiro, v. 9, n. 2, dez. 2000. Disponível em: $<$ http://scielo.iec.pa.gov.br/scielo.php?pid=S0103-

460X2001000200008\&script=sci_arttext>. Acesso em: 20 ago. 2015. 


\section{Artigo Original}

\section{Gestão em Saúde}

2 - Doménech X. Química ambiental: el impacto ambiental de los residuos. 2. ed. Madrid: Miraguano, 1994. 254 p.

3 - Schneider VE et al. Manual de Gerenciamento de Resíduos Sólidos de Serviços da Saúde. 2. ed. Caxias do Sul: Educs, 2004. 319 p.

4 - Brasil. Lei no 12305, de 02 de agosto de 2010. Institui A Política Nacional de Resíduos Sólidos; Altera A Lei no 9.605, de 12 de Fevereiro de 1998; e Dá Outras Providências. Brasília, Disponível em: <http://www.planalto.gov.br/ccivil_03/_ato2007-2010/2010/lei/l12305.htm>. Acesso em: 19 set. 2015.

5 - Associação Brasileira de Normas Técnicas. NBR 12807: Resíduos de Serviços da Saúde: Terminologia. São Paulo: 1993. Disponível em: http://licenciadorambiental.com.br/wp-content/uploads/2015/01/NBR-12.807-

Residuos-de-Servi\%C3\%A7os-de-sa\%C3\%BAde.pdf. Acesso em: 30 set. 2015.

6 - Coelho H. Rejeitos em saúde: como descartar, aproveitar e gerenciar. Rio de Janeiro: Associação Nacional de Biossegurança, 2003. Ano 3, n 10.

7 - Corrêa LB. A educação ambiental e os resíduos sólidos de serviços da saúde: a formação acadêmica.2005. 120 f. Dissertação (Mestrado) - Curso de Educação Ambiental, Universidade Federal do Rio Grande, Rio Grande, 2005. Disponível em: <http://www.argo.furg.br/bdtd/tde_arquivos/5/TDE-2008-06-26T102338Z88/Publico/Bilhalva.pdf>. Acesso em: 12 set. 2015.

8 - Associação Brasileira de Normas Técnicas. NBR 12809: Manuseio dos Resíduos dos Serviços da Saúde. Rio de Janeiro: 1993.

9 - Collins $\mathrm{CH}$, Kennedy DA. Microbiological hazards of occupational needlestick and sharps injuries. Journal of Applied Bacteriology 1987;62:385-402.

10 - Garcia LP, Zanetti-Ramos BG. Gerenciamento dos resíduos de serviços de saúde: uma questão de biossegurança. Cadernos de Saúde Pública, [s.I.], v. 20, n. 3, jun. 2004. Disponível em: <http://www.scielosp.org/scielo.php?script=sci_arttext\&pid=S0102311X2004000300011\&lang=pt>. Acesso em: 12 mar. 2016.

11 - Brasil. Resolução no 358, de 29 de abril de 2005. Dispõe sobre o tratamento e a disposição final dos resíduos dos serviços de saúde e dá outras providências. Brasília, Disponível em: <http://www.mma.gov.br/port/conama/res/res05/res35805.pdf>. Acesso em: 30 set. 2015.

12 - Fortes HM. Gerenciamento de Resíduos Serviços de Saúde. 4. ed. Jardim Alvorada: AgÊncia Transfusional - Hospital UniversitÁrio Júlio Muller, 2004. 18 


\section{Artigo Original}

\section{Gestão em Saúde}

p. Disponível em: <http://www.ufmt.br/ufmt/site/userfiles/file/hujm/Comissoes e Comites/Programa de gerenciamento de resíduos serviços de saúde.pdf>. Acesso em: 19 set. 2015.

13 - Brasil. Resolução nํ 306, de 07 de dezembro de 2004. Dispõe Sobre 0 Regulamento Técnico Para O Gerenciamento de Resíduos de Serviços de Saúde. Brasília.

14 - Maneschy CEA. Plano de Gerenciamento de Resíduos de Saúde do Hubfs. Belém: Universidade Federal do Pará, 2009. Disponível em: <http://www.bettina.ufpa.br/documentos/pgrssatualbettina.pdf>. Acesso em: 19 set. 2015.

15 - Souza DD. Importância do gerenciamento de resíduos de serviço de saúde (GRSS) na estratégia de saúde da família (ESF). Universidade Federal de Minas Gerais. Faculdade de Medicina. Núcleo de Educação em Saúde Coletiva. Governador Valadares, 2011. 31f. Monografia (Especialização em Atenção Básica em Saúde da Família). Disponível em: <https://www.nescon.medicina.ufmg.br/biblioteca/imagem/2780.pdf>. Acesso em: 19 set. 2015.

16 - Bartholomeu DB, Branco JEH, Caixeta Filho JV. Caracterização da Logística de Resíduos de Serviços de Saúde (RSS). In: Bartholomeu DB, Caixeta Filho J V. Logística Ambiental de Resíduos Sólidos. São Paulo: Atlas, 2011. p. 44-66.

17 - Brasil. Ministério da Saúde. SMP como funciona. Brasília: Portal da Saúde. Disponível em: <http://dab.saude.gov.br/portaldab/smp_como_funciona.php>. Acesso em: 09 mar. 2016

18 - Silva CE, Hoppe AE. Diagnóstico dos resíduos de serviços de saúde no interior do Rio Grande do Sul. Engenharia Sanitaria e Ambiental [s.l.] 2005;10(2):146-51.

19 - Alves SB. Manejo de Resíduos de Serviços de Saúde na Atenção Básica. 2010. 145 f. Tese (Doutorado) - Curso de Enfermagem, Universidade Federal de Goiás, Goiânia, 2010. Disponível em: $<$ https://mestrado.fen.ufg.br/up/127/o/Sergiane_Bisinoto_Alves.pdf>. Acesso em: 12 mar. 2016.

20 - Rapparini C, Vitória MAA, Lara LTR. Recomendações para atendimento e acompanhamento de exposição ocupacional a material biológico : HIV e hepatites B e $\quad$ C. Brasil . Disponível em: <http://bvsms.saude.gov.br/bvs/publicacoes/04manual_acidentes.pdf>. Acesso em: 12 mar. 2016. 
Artigo Original

Gestão em Saúde

21 - Oliveira NC, Mouta ERF. Precauções básicas e gerenciamento de resíduos na coleta para o exame de Papanicolaou. Revista da Rede de Enfermagem do Nordeste 2009;10(3):19-26. Disponível em: $<$ http://www.revistarene.ufc.br/revista/index.php/revista/article/view/510/pdf>. Acesso em: 12 mar. 2016.

22 - Almeida VCF et al. Solid waste management in family health units. Northeast Network Nursing Journal 2009;10(2):103-12.

23 - Cervo AL, Bervian PA, Silva R. Metodologia científica. 6. ed. São Paulo: Prentice Hall, 2007.

24 - Vieira CSM. Análise do manejo dos resíduos de serviços de saúde em unidade básica de saúde vinculada a uma Instituição de Ensino Superior. 2013. 78 f. TCC (Graduação) - Curso de Engenharia Ambiental e Sanitária, Universidade Federal de Pelotas, Pelotas, 2013. Disponível em: <http://wp.ufpel.edu.br/esa/files/2013/10/TCC-CATIA-VIEIRA.pdf>. Acesso em: 16 abr. 2016.

25 - OPAS. Organização Pan-Americana de Saúde. Estilos de vida. 1997. 